\title{
DYNAMICAL IMPLICATIONS OF DIFFUSIVE AND CONVECTIVE COSMIC RAY PROPAGATION IN GALACTIC HALOS
}

\author{
D. BREITSCHWERDT ${ }^{1}$, J.F. MCKENZIE ${ }^{2}$ AND H.J. VÖLK ${ }^{1}$ \\ ${ }^{1}$ MPI für Kernphysik, D-6900 Heidelberg, Postfach 103980, FRG \\ ${ }^{2}$ MPI für Aeronomie, D-3411 Katlenburg-Lindau, Postfach 20, FRG
}

\begin{abstract}
On the basis of our present knowledge about Cosmic Ray (CR) propagation, it is argued that galactic halos should mainly consist of two parts, namely a lower region, extending from the disk-halo interface to a few $\mathrm{kpc}$, in which CR diffusion prevails and an upper region, where convection dominates. The upper part is the possible site of galactic wind formation due to the strong coupling of the CRs to the thermal plasma via (mainly outwards propagating) Alfvén waves as a mediator. In the lower halo and also in the disk, the gas will be effectively static, the wave field will be almost random in its direction (due to stochastic gas motions), and the CRs must diffuse through the gas to escape. We present a model that describes both the upper and the lower halo and briefly discuss simple analytic solutions for the diffusion and numerical results for the convection region.
\end{abstract}

\section{INTRODUCTION}

It has been argued that there should be a constant efflux of mass and energy into the halo, presumably powered by supernovae ( $\mathrm{SNe}$ ) and stellar winds ( $\mathrm{SWs}$ ). Qualitatively, for the model presented here, it is not essential whether this occurs in a correlated (Norman, 1990) or a distributed (Kahn, 1990) fashion. The important facts are the existence of hot, ionized gas and CRs, as shown by the observations of diffuse X-rays (Nousek et al., 1982) and "thick" radio disks (Hummel et al., 1984), respectively. Presumably at $|z|>$ few kpc the gas will expand laterally and try to attain pressure equilibrium. Magnetic lines of force will be pushed open by the combined pressure of gas and CRs (cf. Breitschwerdt et al., 1990a; Völk, 1990). The activity of star formation in the disk below and the break-out of shells and/or supershells will provide a sufficient level of turbulence, so that the wave field in the lower halo will be isotropic to lowest order and therefore the ensemble averaged Alfvén speed $\left\langle\mathbf{v}_{\mathbf{A}}\right\rangle \approx 0$. This leaves only diffusion as the mechanism for CRs to propagate into the upper halo. Another observational fact is that the CRs do eventually have to escape from the Galaxy, probably along magnetic field lines pointing away from it. There will then be a resonant growth of waves, caused by 
the CRs streaming along these field lines (self-excited waves). The now dominantly outwards propagating waves will cause a considerable momentum transfer of the CRs to the plasma in $z$ during scattering and thus lead to convective flow in the upper halo, combined with an Alfvénic drift of the CRs.

\section{SIMPLE MODEL FOR THE DIFFUSIVE HALO REGION}

This region extends from the Galactic disk up to the reference level $(0 \leq z \leq 1$ $\mathrm{kpc}), z$ being the coordinate perpendicular to the disk. Since the CR disk has at least a half thickness of $300 \mathrm{pc}$, as inferred from the CR propagation properties, the above value for the reference level should be considered as a lower limit. The hot gas flowing out into the halo is essentially the one swept up by supernova remnants (SNRs) and has a temperature of $T \leq 10^{6} \mathrm{~K}$. The majority of SNRs are the result of Type II SN explosions and are decreasing exponentially by number with a scale height of $H_{\mathrm{SN}} \sim 55 \mathrm{pc}$ (Bregman 1980). Therefore there will be a mass loading of the lower halo with hot gas at a rate $Q(z)$. The simplest representation, reflecting such an exponential decrease of sources is one which is just proportional to the local gas density $\rho$, i.e. $Q(z)=\alpha \rho(z)$, where $\alpha^{-1}$ is a characteristic time scale for the mass loading. The expanding gas will cool adiabatically, but there is also an input of energy by shock heating of Type I SNe, as well as from Type II from runaway $O$ stars. Therefore for $|z| \leq 1 \mathrm{kpc}$, an isothermal equation of state seems convenient and more appropriate. We note that in the momentum equation, there has to appear also a term for the drag force per unit mass $Q u / \rho$ due to the mass loading. Because of $\left\langle\mathbf{v}_{\mathbf{A}}\right\rangle \approx 0$ and small wave pressure, diffusion dominates convection as long as the mass velocity $u$ satisfies $|u| \ll \bar{\kappa} / h_{c}=65 \mathrm{~km} / \mathrm{s}$, where typical values have been adopted for the averaged diffusion coefficient $\bar{\kappa}=10^{29} \mathrm{~cm}^{2} / \mathrm{s}$ and the CR pressure scale height $h_{c} \sim 5 \mathrm{kpc}$. Applying the above assumptions and taking $z$ as the only independent variable, the equations in their steady-state form read:

$$
\begin{aligned}
\frac{d}{d z}(\rho u) & =\alpha \rho, \\
\frac{d}{d z}\left(\rho u^{2}+p_{g}+p_{c}\right) & =-\rho g_{\mathrm{eff}} \\
-\frac{\bar{\kappa}}{\gamma_{c}-1} \frac{d p_{c}}{d z} & =\text { const. } \\
T(z) & =\text { const. }
\end{aligned}
$$

Here $p_{g}, p_{c}, T, g_{\text {eff }}$ and $\gamma_{c}$ denote the gas pressure, the CR pressure, the gas temperature, the effective gravitational acceleration, and the CR adiabatic index (4/3 for an ultra-relativistic gas), respectively. We have shown (Breitschwerdt et al., 1990b) that the specific form of the mass loading term allows for the separation of the variables $\rho$ and $u$, to give

$$
\frac{d^{2} \rho}{d z^{2}}+\frac{2 \alpha^{2}}{c^{2}} \rho+\frac{1}{c^{2}} \frac{d}{d z}\left(\rho g_{\mathrm{eff}}\right)=0
$$


where the isothermal speed of sound is $c^{2}=k_{B} T / \mu$, with $k_{B}$ being Boltzmann's constant and $\mu$ the mean mass per particle. To solve equation (5) we have to impose the following boundary conditions (BCs) at $z=0: \rho=\rho_{d},(d \rho / d z)_{z=0}=$ $-\rho_{d} g_{\mathrm{eff}} / c^{2}+p_{c d} /\left(h_{c} c^{2}\right)$, whereby subscript ' $\mathrm{d}$ ' refers to quantities evaluated in or close to the galactic disk. We note that according to equation (5) and the $\mathrm{BCs}$, there are three different scale heights involved in the problem, viz. $l=c^{2} / g_{\text {eff }}$ (thermal gas), $c / \alpha$ (mass loading) and $h_{c}$ (CRs). Therefore the structure of the lower halo can be quite different from a simple hydrostatic model. To discriminate between the various solutions, it is convenient to define a characteristic dimensionless parameter $\Delta=8 l^{2} \alpha^{2} / c^{2}$. There are three different classes of solutions, according to whether $\Delta<1,=1,>1$, respectively, i.e.:

$$
\begin{aligned}
& \frac{\rho}{\rho_{d}}=\frac{\exp \left(m_{+} z\right)}{\left(m_{-}-m_{+}\right)}\left[m_{-}+\frac{1}{l}-k\right]+\frac{\exp \left(m_{-} z\right)}{\left(m_{+}-m_{-}\right)}\left[m_{+}+\frac{1}{l}-k\right], \\
& \frac{\rho}{\rho_{d}}=\exp (-z / 2 l)\left[1+\left(k-\frac{1}{2 l}\right) z\right], \\
& \frac{\rho}{\rho_{d}}=\exp (-z / 2 l)\left[\cos (\beta z)+\beta^{-1}\left(k-\frac{1}{2 l}\right) \sin (\beta z)\right],
\end{aligned}
$$

where $k=p_{c d} /\left(h_{c} \rho_{d} c^{2}\right), m_{\mp}=-(1 / 2 l)(1 \mp \sqrt{1-\Delta})$ and $\beta=\sqrt{\left(2 \alpha^{2} / c^{2}\right)-\left(1 / 4 l^{2}\right)}$. The flow speed $u$ then follows from integration of the continuity equation (1): $u(z)=(\alpha / \eta) \int_{0}^{z} \eta d z^{\prime}, \eta \equiv \exp \left(-\int H^{-1} d z^{\prime}\right), H^{-1} \equiv-(1 / \rho)(d \rho / d z)$. To illustrate these solutions, we use some typical values for the parameters: $g_{\text {eff }}=10^{-8} \mathrm{~g} / \mathrm{cm}^{2}$ (at a Galactocentric radius $R_{0}=10 \mathrm{kpc}$ ), $T=10^{6} \mathrm{~K}$, and $\alpha$ we estimate to be the ratio of the total mass loss rate into the halo to the total halo mass, i.e. $\dot{\mathcal{M}}_{H} / \mathcal{M}_{H}$. Both of these numbers are highly uncertain and $\dot{\mathcal{M}}_{H}$ varies from $2.1 M_{\odot} / \mathrm{yr}$ to $22 M_{\odot} / \mathrm{yr}$ (cf. Heiles, 1987), while $\mathcal{M}_{H}$ is supposed to lie between $7 \times 10^{7} M_{\odot}$ (Bregman, 1980) and $9.8 \times 10^{7} M_{\odot}$ (Heiles, 1987). Taking the lower values we get: $\alpha=9.5 \times 10^{-16} \mathrm{~s}^{-1}, l=1.65 \times 10^{22} \mathrm{~cm}$ and $\Delta=11.6$. Since $\beta z<1$ we can approximate equation (6c) to obtain $\rho / \rho_{d}=\exp (-z / 2 l)\left[1-(z / 2 l)+p_{c d} z /\left(\rho_{d} c^{2} h_{c}\right)\right]$. For $p_{c d}<\rho_{d} c^{2}$ we get $\rho_{0}=\rho(z=1 \mathrm{kpc})=0.8 \rho_{d}$. Because of the uncertainties in the parameters and since $\Delta$ is not too different from unity, each of the solutions $(6 a)$ (6c) may be relevant in practice.

\section{TRANSITION TO THE CONVECTIVE HALO REGION}

At distances $|z| \geq 1 \mathrm{kpc}$ from the disk, the dominant source of waves will be selfexcitation, and we then expect Alfvénic drift to be so efficient, that convection will dominate diffusion in the CR transport; at least this is our assumption for the sequel. The change in geometry, from plane parrallel to spherically symmetric flow, as $|z|$ increases has been modelled by a simple flux tube geometry. All this has been described in detail by Breitschwerdt et al. $(1987,1990 \mathrm{a})$; we will therefore present 
only the most important results. The model calculations have been carried out for a flux tube located at $R_{0}=10 \mathrm{kpc}$ and a set of paramters given at reference level $|z|=1 \mathrm{kpc}: \rho_{0}=1.67 \times 10^{-27} \mathrm{~g} / \mathrm{cm}^{3}, p_{g}=2.8 \times 10^{-13} \mathrm{dyne} / \mathrm{cm}^{2}, p_{c}=$ $1.0 \times 10^{-13}$ dyne $/ \mathrm{cm}^{2}, B_{0}=1 \mu \mathrm{G}$ for the magnetic field strength in $|z|$-direction and a mean fluctuating field component $\langle\delta B\rangle=0.1 B_{0}$. It turns out that the initial mass velocity is quite low, $u_{0}=10 \mathrm{~km} / \mathrm{s}$, and that the flow accelerates with increasing $|z|$, attaining an asymptotic value of $u_{f}=310 \mathrm{~km} / \mathrm{s}$. We emphasize that the low base velocity $u_{0}$ (subalfvénic) is consistent with the flow properties derived for the lower halo and therefore guarantees a continuity of the two types of solutions.

\section{CONCLUSIONS}

The presence of CRs in galactic halos is important, both for its density structure and its dynamics. In particular, we have shown that the propagation properties of the CRs, in a simple minded picture, give rise to two essentially different, i.e. diffusive and convective, regions which can be connected in a smooth fashion. Since there is much more observational information about the lower halo, it is certainly worth while incorporating more physics into our simple model, such as momentum addition, heating and cooling and linear wave damping. So far, we can only assess that if the CRs mainly diffuse through the halo, their support of the plasma against gravity due to their large scale height is rather weak. However, there is also mass loading from the sources, which modifies the thermal scale height. Once Alfvénic drift becomes important, the plasma is accelerated considerably against the drag of the gravitational field and the "overburden" will be lifted to infinity (galactic wind).

\section{REFERENCES}

Bregman, J.N. (1980) Astrophys. J. 236, 577

Breitschwerdt, D., McKenzie, J.F., Völk, H.J. (1987) in "Interstellar Magnetic Fields", Beck, R., Gräve, R. (eds.), Springer-Verlag, Heidelberg, p. 131

Breitschwerdt, D., McKenzie, J.F., Völk, H.J. (1990a) Astron. Astrophys. (submitted)

Breitschwerdt, D., McKenzie, J.F., Völk, H.J. (1990b) Proc. 21st Int. Cosmic Ray Conf. (Adelaide) 3, 315

Heiles, C. (1987) Astrophys. J. 315, 555

Hummel, E., Sancisi, R., Ekers, R.D. (1984) Astron. Astrophys. 133, 1

Kahn, F.D. (1990) these proceedings

Norman, C.A. (1990) these proceedings

Nousek, J.A., Fried, P.M., Sanders, W.T., Kraushaar, W.L. (1982) Astrophys. J. 258, 83

Völk, H.J. (1990) these proceedings 